\title{
Precision measurements of gravity using cold atom sensors
}

\section{F. Sorrentino}

\section{M. de Angelis}

marella.deangelis@fi.infn.it

\section{A. Bertoldi}

\section{Cacciapuoti}

\section{A. Giorgini}

\section{Prevedelli}

\section{G. Rosi}

\section{G.M. Tino}

\author{
Istituto di Cibernetica CNR, via Campi Flegrei 34, I-80078 Pozzuoli (NA) Italy \\ Dipartimento di Fisica, Universita' di Firenze - INFN, via Sansone 1 Polo Scientifico, \\ I-50019 Sesto Fiorentino (Firenze) Italy \\ Istituto di Cibernetica CNR, via Campi Flegrei 34, I-80078 Pozzuoli (NA) Italy \\ Laboratoire Charles Fabry de l'Institut d'Optique, CNRS, Campus Polytechnique, RD 128, \\ F-91127 Palaiseau Cedex, France \\ ESA Reasearch and Scientific Support Department, ESTEC, Keperlaan 1 - P.O. Box 299, \\ 2200 AG Noordwijk ZH, The Netherlands \\ Dipartimento di Fisica, Università di Napoli, via Cinthia, I-80126, Italy. \\ Dipartimento di Chimica Fisica e Inorganica, Università di Bologna, V.le Risorgimento 4, \\ I-40136 Bologna, Italy \\ Dipartimento di Fisica "Enrico Fermi" - Largo Bruno Pontecorvo 3, I-56127 Pisa, Italy
}

Dipartimento di Fisica and LENS, Università di Firenze - INFN, via Sansone 1 Polo Scientifico, I-50019 Sesto Fiorentino (Firenze) Italy

We present a synthetic view of experiments we are performing using atom interferometry to determine the gravitational constant $G$ and to test the Newtonian gravitational law at micrometric distances. Accurate gravity measurements with atom interferometry also find applications in geophysical studies and in satellite missions for the geoid mapping. Experiments in progress, using ultracold atom devices, for applications in geophyiscal and space monitoring will be also described. [D0l: 10.2971/je05.2009.09025]

Keywords: atom sensors, gravity measurements, environmental and space applications

\section{INTRODUCTION}

Matter-wave interferometry has recently led to the development of new techniques for the measurement of inertial forces, finding important applications both in fundamental physics and in applied research. The remarkable stability and accuracy that atom interferometers have reached for acceleration measurements can play a crucial role for gravimetry. Atom interferometry is used for precise measurements of gravity acceleration [1], Earth's gravity gradient [2, 3] and rotations [4]. Accelerometers based on atom interferometry have been developed for many practical applications including metrology, geodesy, geophysics, engineering prospecting and inertial navigation. Ongoing studies show that the space environment will allow to take full advantage of the potential sensitivity of atom interferometers [5].

Precision gravity measurements by atom interefrometry are becoming important in many metrological applications. For the Newtonian constant $G$, neutral atoms are used as microscopic test masses to probe the gravitational field generated by a well characterized source mass $[2,6,7]$. The new definition of the unity of mass (the kilogram) depending on the Planck's constant $h$ is also attracting increasing interest. The link will be realised by means of a Watt balance, which will compare an electric to a mechanical power. In this scheme an accurate measurement of the acceleration of gravity is needed, that can be attained by the atom interferometer sensor proposed in $[8,9]$.

Exotic theories predict deviations of the Newtonian gravitational potential at small distances (in the range $<1 \mathrm{~mm}$ ). To test these possible deviations a sample of ultracold bosonic ${ }^{88} \mathrm{Sr}$ atoms loaded into a vertical optical standing wave has been used.

In this paper we present the general principles of operation of atom interferometers. Future perspectives for accurate gravity measurement aiming to environmental monitoring and space applications are also presented.

\section{ATOM SENSOR PRINCIPLES}

The de Broglie wavelength of an atom matter-wave is $\lambda_{m w}=$ $h / M v$, where $h$ is the Plank constant, $M$ the atom mass and $v$ its velocity. For cold atoms with temperature of $\simeq 1 \mu \mathrm{K}$, this 
wavelength may be comparable to $1 \mu \mathrm{m}$. In the following the principles of the Raman atom interferometer technique and the technique based on atom Bloch oscillations in an optical periodic potential will be described.

In the first experiments that demonstrated the potential of atom interferometry for accurate measurements of the Earth's gravity acceleration [1] and its gravity gradient [2,3], the separation and reflection of the atomic wave packet is achieved by using Raman atom interferometry, that is by inducing a Raman transition between internal states of the atoms by an electromagnetic field [10]. Let us consider an alkali atom as a two-level system, represented by the two hyperfine states 1 and 2 of the ground level. Two counter-propagating beams, with propagation vectors $\mathbf{k}_{\mathbf{1}}$ and $\mathbf{k}_{2} \simeq-\mathbf{k}_{1}$, whose frequency difference is resonant with the atomic two-level system, drive a two-photon Raman transition between the two hyperfine states 1 and 2. The internal state of the atom and its momentum are always coupled, as a consequence of momentum and energy conservation in the system atom+photons. During the flight, optical pulses are used to stimulate Raman transitions between two different hyperfine states. These light pulses act on the atom matter-waves as mirrors and beamsplitters act on the light-wave in an optical interferometer. In the case the velocity of the atoms is collinear with the effective Raman wavevector, the atomic phase shift measured with the interferometer is sensitive to the linear acceleration of the atoms. The resulting phase difference accumulated along the atom interferometer paths is given by

$$
\Delta \varphi=\mathbf{k}_{e f f} \cdot \mathbf{a} T^{2}
$$

whenever a constant acceleration $\mathbf{a}$ is present and where $\mathbf{k}_{\text {eff }}=\mathbf{k}_{2}-\mathbf{k}_{1} \simeq-2 \mathbf{k}_{1}$ and $T$ is the temporal separation between two optical pulses (see Figure 1).

As an alternative technique, artificial crystals of light, consisting of hundreds of thousands of optical microtraps, are created by interference of two counter-propagating laser beams. When a sample of atoms interacts with such a laser standing wave, the standing wave acts as a diffraction grating for the atoms. Under the influence of such a periodic potential and a weak uniform force, the atomic momentum changes periodically across the first Brillouin zone, a phenomenon known as Bloch oscillations [11]. Bloch oscillations of an atom in a standing wave are directly related to the uniform force and thus to $g$ : the combination of the periodic optical potential and the linear gravitational potential gives rise to Bloch oscillations at a frequency $v_{B}=m g \lambda_{L} / 2 h$, where $m$ is the atomic mass, $g$ is the acceleration of gravity, $\lambda_{L}$ is the wavelength of the light producing the lattice, and $h$ is the Planck constant. Since $\lambda_{L}$, $m$, and $h$ are well known, the acceleration along the lattice axis can be determined by measuring the Bloch frequency $v_{B}$.

\section{MEASUREMENT OF GRAVITATIONAL CONSTANT G}

In metrology the determination of the gravitational constant is one of the oldest and most intriguing problems. The CODATA-2006 recommended value for $G$ is $6.67428(67) \times 10^{-11} \mathrm{~m}^{3} \mathrm{~kg}^{-1} \mathrm{~s}^{-2}$ [12]. $G$ is still affected by an
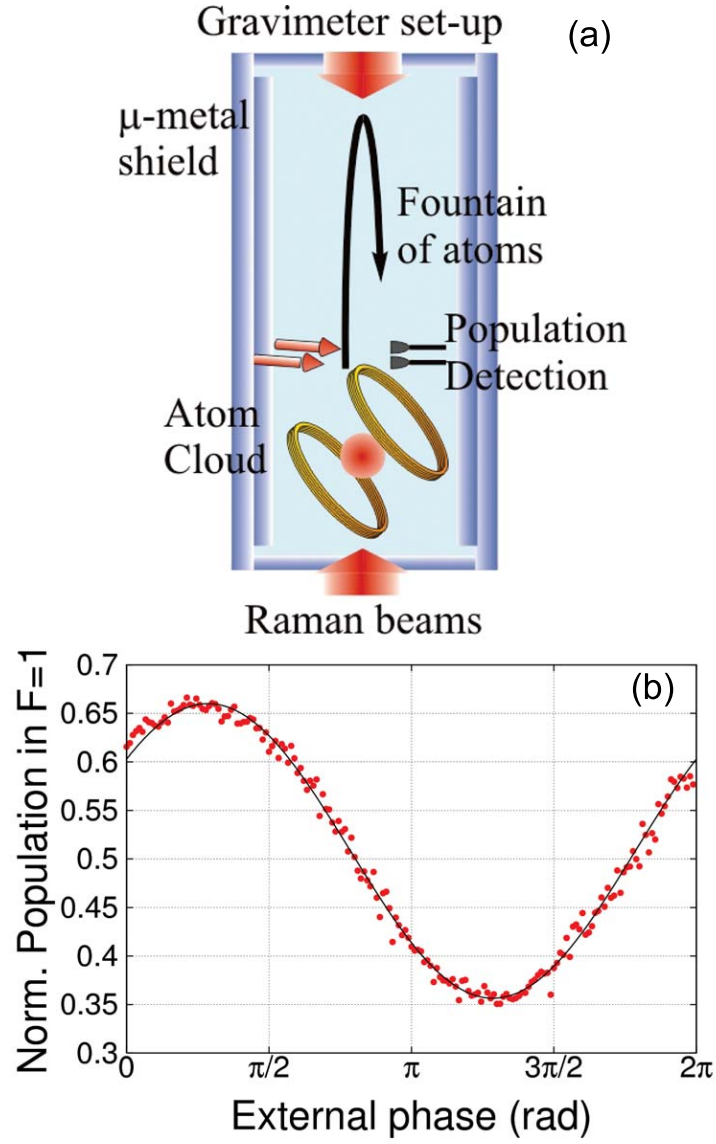

FIG. 1 Experimental setup of an atom interferometer and typical interference fringes obtained by scanning the relative phase of the Raman lasers. The atom fringes have been recorded in the gravity experiment in Firenze [2]. Experiment interrogation time between the light pulses is $T=5 \mathrm{~ms}$.

uncertainty of $100 \mathrm{ppm}$, much higher than that of any other fundamental constant, and the results of most recent experiments differ by several hundreds ppm. Among the possible sources for this unexplained discrepancy one in particular appears mostly critical. The majority of the experiments performed so far are based on macroscopic suspended masses. Systematic effects and parasitic couplings in suspending fibers could be responsible for the observed discrepancies.

An experiment for measuring the gravitational acceleration induced by moving masses is proposed in $[2,7]$, where freely falling rubidium atoms are used as probes of the gravitational field induced by a well modelled source mass. From the differential acceleration measurements and from the knowledge of the added mass distribution, it is possible to determine the value of $G$ (see Figure 2). The experiment is designed to control systematic errors below the $10^{-4}$ level. In that experiment two sets of source masses are symmetrically arranged in a cylindrical geometry around the vacuum tube where atoms are freely falling, and can be vertically moved with high precision. They are positioned close to the atomic trajectories and generate a well known gravitational field. For the determination of $G$, the source masses signal is detected as variation on the gradient of gravity acceleration. If $g_{D W}$ and $g_{U P}$ are the gravity acceleration values at the height of the lower and upper interferometers the relative phase shift is $\Delta \varphi=$ $k_{e f f}\left(g_{D W}-g_{U P}\right) T^{2}$. For the determination of $G$ a double dif- 
ferential scheme is implemented. The measurements are repeated twice with atom clouds in the same point, but with the two source masses in different positions. In this way all contributions that are constant in time during the measurement cycle, like rotations or gradient contribution, are suppressed. The change of the local acceleration due to the added gravitational potential can be measured, thus allowing determining the gravitational constant $G$, once the source masses density distribution and their positions are well known. In Figure 2 interferometer data distributed along ellipses are shown. The data are obtained by plotting the readout of the upper interferometer versus the readout of the lower interferometer and recorded with source masses in two different positions with respect to the atomic sensors: red points are obtained with the two sets of source masses one close to the other and in between the two atom interferometers, blue points, instead, by moving the two sets of masses far apart. The differential ellipticity in the two configurations is the signal from which $G$ is determined [13].
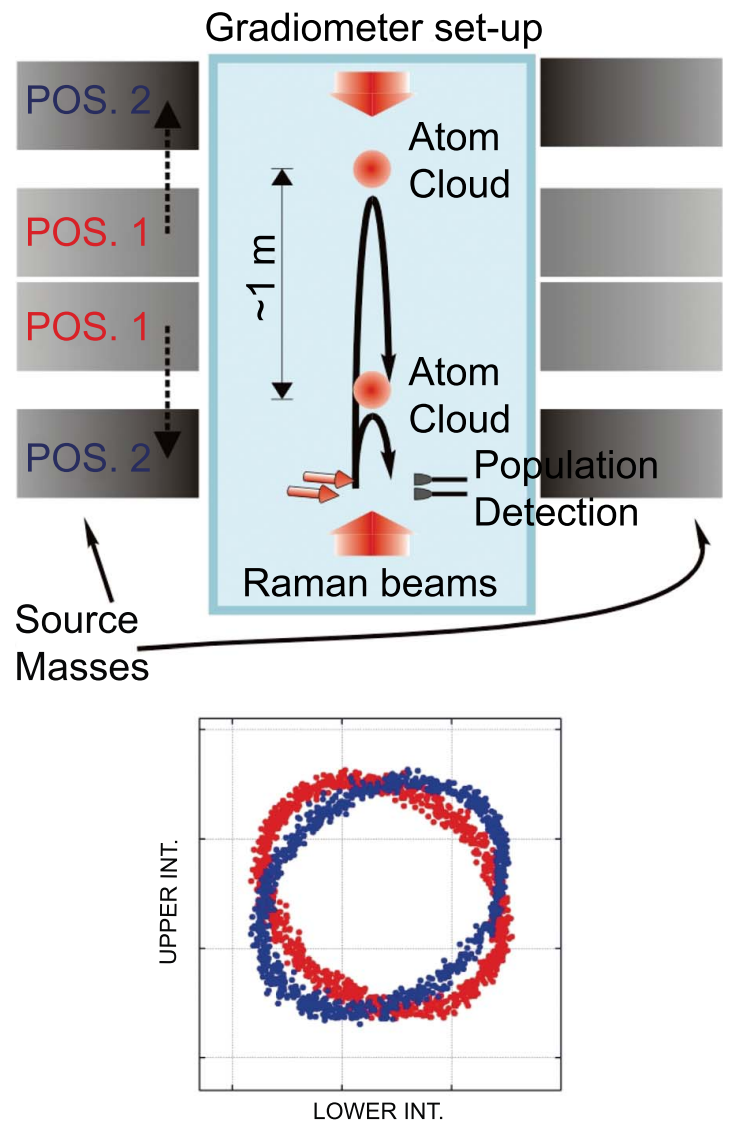

\section{Ellipses for two positions of source masses}

FIG. 2 Scheme of the gravity gradiometer used in Firenze for the measurement of $G$ [2]. The differential ellipticity in the two configurations is the signal from which $G$ is determined.

\section{SMALL SPATIAL SCALE MEASUREMENT}

The possible deviations from the Newtonian gravitational potential are usually described by adding a Yukawa-type term

$$
V(r)=-G \frac{m_{1} m_{2}}{r}\left(1+\alpha e^{-r / \lambda}\right),
$$

where $G$ is the Newtonian gravitational constant, $m_{1}$ and $m_{2}$ are the masses, and $r$ is the distance between them. The parameter $\alpha$ gives the relative strength of deviations from Newtonian gravity and $\lambda$ is its spatial range. Experiments searching for possible deviations have set bounds for the parameters $\alpha$ and $\lambda$. Theories predict deviations in the range where $\lambda<1 \mathrm{~mm}$. Recent results using micro-cantilever detectors lead to extrapolated limits $\alpha \sim 10^{4}$ for $\lambda \sim 10 \mu \mathrm{m}$ [14], while for distances below $10 \mu \mathrm{m}$ it was not possible to perform direct experiments so far.

The confinement of ultracold atoms in optical lattices provides clean model systems to study deviation of the Newtonian law at short distances. The small size and high sensitivity of the atomic probe allow a model-independent measurement at distances of a few $\mu \mathrm{m}$ from the source mass, giving direct access to unexplored regions in the $\alpha-\lambda$ plane, like in [11], where laser-cooled ${ }^{8} \mathrm{Sr}$ atoms are trapped in a 1-dimensional vertical optical lattice. In this experiment the combination of the periodic optical potential and the linear gravitational potential gives rise to Bloch oscillations. From the measured Bloch frequency $v_{B}$ the gravity acceleration along the optical lattice is estimated with a sensitivity of $4 \times 10^{-6} g$ at $1 \mathrm{~s}$. Although the sensitivity is much lower than that achieved with other atom interferometry sensors, the small spatial extension of the atomic cloud and the possibility to load it into the optical potential at micrometric distance from a surface makes this scheme particularly suitable for the investigation of forces at small spatial scales (see Figure 3). Preliminary tests suggest that such system may allow to directly test non-Newtonian gravity in the region around $\lambda \sim 5 \mu \mathrm{m}$ with a sensitivity to the $\alpha$ value of $10^{3}$, more than one order of magnitude lower than the present bounds [11].

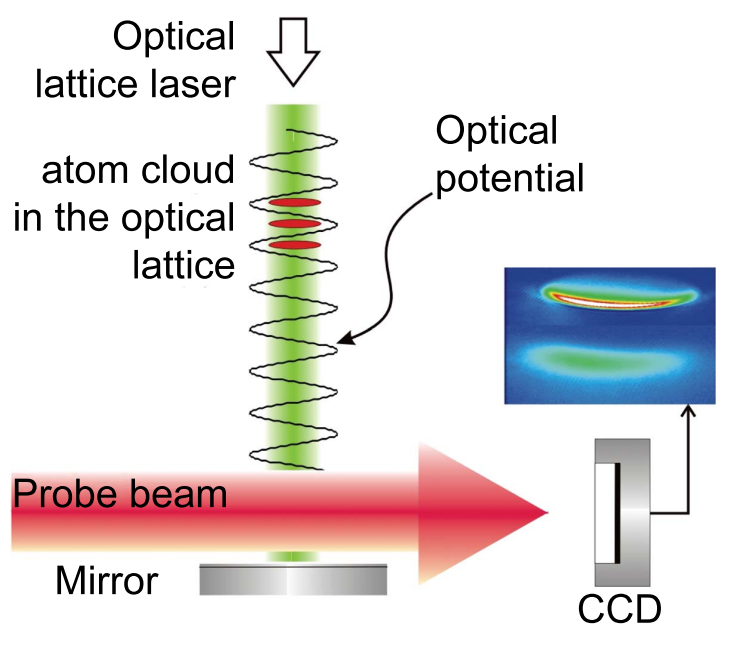

FIC. 3 Experimental set-up for producing Bloch oscillations of atoms in a light standing wave [11].

\section{ATOM INTERFEROMETER SENSORS APPLICATIONS}

Accurate gravity measurements find applications in many fields of physics: in metrology for both the definition of SI units and a better knowledge of fundamental constants; in high-precision test of general relativity, in satellite gravity missions for the geoid mapping, and in geophysical stud- 
ies. Users' interest in terrestrial gravity measurements are in high resolution prospecting for resource exploration, monitoring of man-induced processes, high resolution geoid mapping (or positioning), investigation of subsurface mass redistributions at local (e.g. volcanoes and geothermal systems), regional (post-glacial rebound) and global scales (Earth's mantle and core). Data from gravity surveys at the Earth's surface are usually processed relatively to a network of reference stations. The systems of stations show an accuracy decay during time, which is mostly due to decay of the relative instruments (like spring gravimeter) used there. For such stations, instrument accuracy needs to be constantly monitored. Recently, continuous micro-gravimetric observations have found a further, important field of application to volcano monitoring. It appears that mass redistribution in volcanic systems occur over time scales spanning the $10^{2}-10^{6} \mathrm{~s}$ interval (i.e. minutes to a month), and have amplitudes ranging from $10 \mu \mathrm{gal}$ up to hundreds of $\mu \mathrm{gal}\left(1 \mu \mathrm{gal}=10^{-9} \mathrm{~g}\right)$, which means that a long term stability instrument is needed to have a resolution of $1-10 \mu \mathrm{gal}$ over 1 year. Subsurface mass redistributions of geodynamical interest occur over time scales, spanning the $10^{6}-10^{8} \mathrm{~s}$ interval (i.e. month to years) and produce gravity variations whose amplitude ranges from a few microgals up to hundreds of microgals. Although subtle, these variations generally precede other surface-detectable signals such as strain deformations and/or seismic ground shaking. Taken all together, these considerations indicate that the continuous observation of the local gravity field using sensitive instrumentation with $\mu$ gal accuracy and over long periods (years) is a major goal to be attained toward a better understanding of geodynamic processes and a successful assessment of earthquake and volcanic hazards. For long period stable measurements a new portable gravimeter is under construction within the European project FINAQS (Future Inertial Atomic Quantum Sensors); the instrument aims to an accuracy in the range of 1 parts in $10^{10}$ [15], an improvement of one order of magnitude over the best current instruments. Satellite missions are presently used to map the geoid. In these missions the geopotential second order derivatives, i.e. the relative accelerations of proof masses, are measured along the orbit. The second order derivatives are then used in an inverse problem to calculate the gravitational potential. The ESA gravity mission GOCE [16], is based on a gravity gradiometer. It is currently believed that a sensor for any GOCE follow-on mission cannot be obtained with presently available three-axis gravity gradiometers concepts. Atom interferometry represents a promising technology. Good differential acceleration sensitivities have been already demonstrated on ground and a significant improvement can be foreseen for operation in space on a satellite in geodetic motion. A space gravity gradiometer in microgravity conditions where interrogation time up to $10 \mathrm{~s}$ (see Eq.(1)) could in principle achieve a sensitivity better than $10^{-12} \mathrm{~s}^{-2}$ at $0.1 \mathrm{~Hz}$.

\section{CONCLUSION}

Atom interferometry represents a promising technology for precision measurements related to gravity. In this paper we have presented atom interferometry technique for a measurement of the gravitational constant $G$. This sensor will achieve a precision approaching 1 part in $10^{4}$. We have also presented the possibility to test the deviations at short distances of the gravitational potential, using a sample of ultracold bosonic ${ }^{88} \mathrm{Sr}$ atoms to observe Bloch oscillations. The small size of the system enables precision measurements of forces at micrometer scale to study deviations from Newtonian gravity predicted by theories beyond the standard model.

Atom interferometry is also a promising technique for environmental gravity monitoring and for gravity space missions. Accurate and portable gravimeters based on atom interferometry are now feasible and can be used for geophysical application where stable monitoring over long periods (years) is needed. In atom interferometers good differential acceleration sensitivities have been already demonstrated on ground and a significant improvement can be foreseen for operation in space on a satellite in geodetic motion, although significant efforts are still ongoing towards a space instrument.

Authors acknowledge financial support from INGV, INFN, ESA, ASI, EC (under FINAQS project no.012986) and CNR (under ESF-Eurocores EUROQuasar-IQS project).

\section{References}

[1] A. Peters, K. Y. Chung, and S. Chu, "Measurement of gravitational acceleration by dropping atoms" Nature 400, 849-852 (1999).

[2] G. Lamporesi, A. Bertoldi, L. Cacciapuoti, M. Prevedelli, and G. M. Tino, "Determination of the Newtonian Gravitational Constant Using Atom Interferometry" Phys. Rev. Lett. 100, 050801 (2008).

[3] J. M. McGuirk, G. T. Foster, J. B. Fixler, M. J. Snadden, and M. A. Kasevich, "Sensitive absolute-gravity gradiometry using atom interferometry" Phys. Rev. A 65, 033608 (2002).

[4] T. L. Gustavson, A. Landragin, and M. A. Kasevich, "Rotation sensing with a dual atom-interferometer Sagnac gyroscope" Classical Quant. Grav. 17, 2385-2398 (2000).

[5] G. M. Tino, L. Cacciapuoti, K. Bongs, Ch.J. Bordé, P. Bouyer, H. Dittus, W. Ertmer, A. Görlitz, M. Inguscio, A. Landragin, P. Lemonde, C. Lammerzahl, A. Peters, E. Rasel, J. Reichel, C. Salomon, S. Schiller, W. Schleich, K. Sengstock, U. Sterr, and M. Wilkens et. al. "Atom interferometers and optical atomic clocks: New quantum sensors for fundamental physics experiments in space" Nucl. Phys. B 166, 159-165 (2007).

[6] J. B. Fixler, G. T. Foster, J. M. McGuirk, and M. A. Kasevich, “Atom Interferometer Measurement of the Newtonian Constant of Gravity" Science 315, 74-77 (2007).

[7] G. Lamporesi, A. Bertoldi, A. Cecchetti, B. Duhlach, M. Fattori, A. Malengo, S. Pettorruso, M. Prevedelli, and G. M. Tino, "Source mass and positioning system for an accurate measurement of $G$ " Rev. Sci. Instrum. 78, 075109 (2007).

[8] P. Cheinet, F. Pereira Dos Santos, T. Petelski, A. Clairon, N. Dimarcq, D. Holleville, and A. Landragin, Cold Atom Absolute Gravimeter for the Watt Balance 60-61 (Conference on Precision Electromagnetic Measurements Digest, 2004).

[9] J. Le Gouët, T. E. Mehlstäubler, J. Kim, S. Merlet, A. Clairon, A. Landragin, and F. Pereira Dos Santos, "Limits to the sensitivity of a low noise compact atomic gravimeter" Appl. Phys. B 92, 133-144 (2008).

[10] K. Moler, D. S. Weiss, M. Kasevich, and S. Chu, "Theoretical anal- 
ysis of velocity-selective Raman transitions" Phys. Rev. A 45, 342348 (1992).

[11] G. Ferrari, N. Poli, F. Sorrentino, and G. M. Tino, "Long-Lived Bloch Oscillations with Bosonic Sr Atoms and Application to Gravity Measurement at the Micrometer Scale" Phys. Rev. Lett. 97, 060402 (2006).

[12] P. J. Mohr, B. N. Taylor, and D. B. Newell "CODATA Recommended Values of the Fundamental Physical Constants: 2006" Rev. Mod. Phys. 80, 633 (2008).

[13] G. T. Foster, J. B. Fixler, J. M. McGuirk, and M. A. Kasevich, "Novel method of phase extraction between coupled atom interferome- ters using ellipse-specific fitting" Opt. Lett. 27, 951-953 (2002).

[14] D. M. Weld, J. Xia, B. Cabrera, and A. Kapitulnik, "A New Apparatus for Detecting Micron-Scale Deviations from Newtonian Gravity" Phys. Rev. D 77, 062006 (2008).

[15] See contributions in http://www.finaqs.uni-hannover.de (2009).

[16] M. R. Drinkwater, R. Haagmans, D. Muzi, A. Popescu, R. Floberghagen, M. Kern, and M. Fehringer, The GOCE gravity mission: ESA's first core Earth explorer volume 62, 1-8 (Proceedings of 3rd International COCE User Workshop, 6-8 November, 2006, Frascati, Italy, 2007). 